\title{
电解制备二氧化锰强酸性电解液中气体扩散电极的稳定性与失效行为
}

\author{
张 慧 ${ }^{1}$ 孟惠民 ${ }^{1,2, *}$
}

('北京科技大学腐蚀和防护中心, 北京 100083 ; ' ${ }^{2}$ 北京科技大学, 北京市腐蚀、磨蚀与表面技术重点实验室, 北京 100083)

摘要: 采用气体扩散电极(GDE)代替传统析氢阴极电解制备二氧化锰(EMD), 重点研究了气体扩散电极在强 酸性 $\mathrm{MnSO}_{4}-\mathrm{H}_{2} \mathrm{SO}_{4}$ 电解液中的稳定性、寿命及失效行为. 结果表明: 气体扩散电极在 $\mathrm{MnSO}_{4}-\mathrm{H}_{2} \mathrm{SO}_{4}$ 电解液中重 现性好、具有一定的稳定性, 寿命可达 $400 \mathrm{~h}$; 平行实验表明, 阳极沉积一定厚度的EMD 是槽电压第一次升高的 主要原因; 电流密度为 $100 \mathrm{~A} \cdot \mathrm{m}^{-2}$ 时, 气体扩散电极失效前阴极过程的速度由氧的离子化反应和氧的扩散混合 控制, 失效后阴极过程由氧去极化和氢去极化共同组成, 主要发生析氢反应; 催化层聚四氟乙烯(PTFE)网络结 构的破坏和镍网层的溶解是电极失效的原因之一; Pt 的团聚降低了电极的电催化活性, 是电极失效的主要原 因; 阴极失效是槽电压再次升高的主要原因.

关键词：气体扩散电极；电解二氧化镇；强酸性电解体系；稳定性；失效 中图分类号： O646; TQ151.4+1

\section{Stability and Failure Behavior of Gas Diffusion Electrodes in Strong Acid Media for Electrolytic Manganese Dioxide}

\author{
ZHANG Hui ${ }^{1} \quad$ MENG Hui-Min ${ }^{1,2, *}$ \\ ( ${ }^{1}$ Corrosion and Protection Center, University of Science and Technology Beijing, Beijing 100083, P. R. China; \\ ${ }^{2}$ Beijing Key Laboratory for Corrosion, Erosion and Surface Technology, \\ University of Science and Technology Beijing, Beijing 100083, P. R. China)
}

\begin{abstract}
Electrolytic manganese dioxide (EMD) was prepared using a gas diffusion electrode (GDE) instead of a traditional hydrogen evolution cathode. The stability, lifetime, and failure behavior of the GDE were studied in a strongly acidic $\mathrm{MnSO}_{4}-\mathrm{H}_{2} \mathrm{SO}_{4}$ electrolysis system. The results show that the GDE has good reproducibility and stability, and its lifetime is up to $400 \mathrm{~h}$ in a $\mathrm{MnSO}_{4}-\mathrm{H}_{2} \mathrm{SO}_{4}$ electrolysis system. Parallel experiments indicate that the major reason for the first increase in the anode cell voltage is the deposition on the anode of a certain thickness of EMD. When the current density is $100 \mathrm{~A} \cdot \mathrm{m}^{-2}$, the cathode reaction rate is controlled by a mixture of oxygen ionization and oxygen diffusion before failure of the GDE, and the cathode reaction process consists of two simultaneous reactions after failure of the GDE, i.e., oxygen depolarization and hydrogen depolarization. Hydrogen depolarization is the main controlling process after GDE failure. One of the reasons for electrode failure is destruction of the polytetrafluoroethylene (PTFE) network structure in the catalyst layer and dissolution of the nickel mesh layer. Platinum agglomeration reduces the electrocatalytic activity of the GDE, and this is the main reason for electrode failure. Cathode failure is the main reason for the second increase in the anode cell voltage.
\end{abstract}

Key Words: Gas diffusion electrode; Electrolytic manganese dioxide; Strong acid electrolysis system; Stability; Failure

Received: June 7, 2013; Revised: October 16, 2013; Published on Web: October 21, 2013.

"Corresponding author. Email: menghm16@126.com; Tel: +86-10-62332548.

The project was supported by the National Natural Science Foundation of China (51274027).

国家自然科学基金(51274027)资助项目

(c) Editorial office of Acta Physico-Chimica Sinica 


\section{1 引言}

我们先前的研究 ${ }^{1,2}$ 阐述了采用气体扩散电极 (GDE)代替析氢阴极用于生产电解二氧化锰(EMD) 的节能减排新方法, 称之为 “氧阴极电解制备二氧 化锰新方法”. 自 1965 年培根电极在阿波罗飞船上 的成功应用, GDE 被推广用于电池系统. 此后, 气体 扩散电极得到了广泛的研究并用以提高电化学反 应的效率. ${ }^{3-5}$ 目前 GDE 主要应用在燃料电池、氯碱 工业、合成过氧化氢等方面, 其中研究环境主要是 碱性、中性和弱酸性环境. ${ }^{6-20}$ 而传统的使用析氢阴 极工业生产电解二氧化锰的电解液体系均为强酸 性体系, 如 $\mathrm{MnSO}_{4}-\mathrm{H}_{2} \mathrm{SO}_{4}$ 电解液的 $\mathrm{pH}$ 值仅为 0.72.1. 可以预见, 气体扩散电极在强酸性电解液中电 解行为和失效行为与碱性和中性电解液体系中的 行为规律不可能完全相同, 但其行为规律的基础研 究基本空白.

为此, 本论文通过电解制备 EMD 强酸性电解 液中气体扩散电极的寿命测试, 失效前后电化学行 为、催化层成分和表面形貌分析, 研究了氧阴极法 制备电解二氧化锰时气体扩散电极在强酸性 $\mathrm{MnSO}_{4}-\mathrm{H}_{2} \mathrm{SO}_{4}$ 电解体系中的稳定性与失效行为, 为 进一步提高气体扩散电极的性能提供理论依据.

\section{2 实验部分}

$\mathrm{Pt} / \mathrm{C}$ 型气体扩散电极主要由催化层和防水透气 层组成, 催化层主要成分有 $\mathrm{Pt} / \mathrm{C}(40 \%)$ 、活性炭 (AR)、乙炔黑 $(50 \%)$ 、聚四氟乙烯(PTFE) (60\%), 防水 透气层主要成分有无水硫酸钠 $(\mathrm{AR})$ 、活性炭、乙炔 黑、PTFE $(60 \%)$ 、镍网. 将催化层和防水透气层组 合, 在一定条件下压成薄片做成氧阴极, 结构示意 图如图 1(a)所示.

阴极采用自制的 $\mathrm{Pt} / \mathrm{C}$ 型气体扩散电极 $\left(5 \mathrm{~cm}^{2}\right)$, 阳极采用钛基钛镇涂层阳极板 $\left(7 \mathrm{~cm}^{2}\right)$, 极间距 5 $\mathrm{cm}$, 组成了氧阴极电解 $\mathrm{MnO}_{2}$ 新方法的气液电解槽, 如图 1(b) 所示. ${ }^{2}$ 电解液为目前工业 EMD 生产常用 的 $120 \mathrm{~g} \cdot \mathrm{L}^{-1} \mathrm{MnSO}_{4} \cdot \mathrm{H}_{2} \mathrm{O}(\mathrm{AR})+30 \mathrm{~g} \cdot \mathrm{L}^{-1} \mathrm{H}_{2} \mathrm{SO}_{4}(\mathrm{AR})$ 强酸性体系, 电解温度为 $70{ }^{\circ} \mathrm{C}$.

本文实验采用的电化学工作站为上海辰华公 司生产的 CHI660B 电化学工作站, 采用三电极体系 测试, 待测电极在电解液中浸泡 $4 \mathrm{~h}$ 后进行测试, 参 比电极采用饱和硫酸亚录 $\left(\mathrm{Hg} / \mathrm{Hg}_{2} \mathrm{SO}_{4}\right)$ 参比电极, 扫 描速率为 $5 \mathrm{mV} \cdot \mathrm{s}^{-1}$. 采用日本理学 $\mathrm{D} / \mathrm{max} 2000 \mathrm{PC} \mathrm{X}$ 射线衍射仪分析电解产物的成分及相结构、电解前 (a)
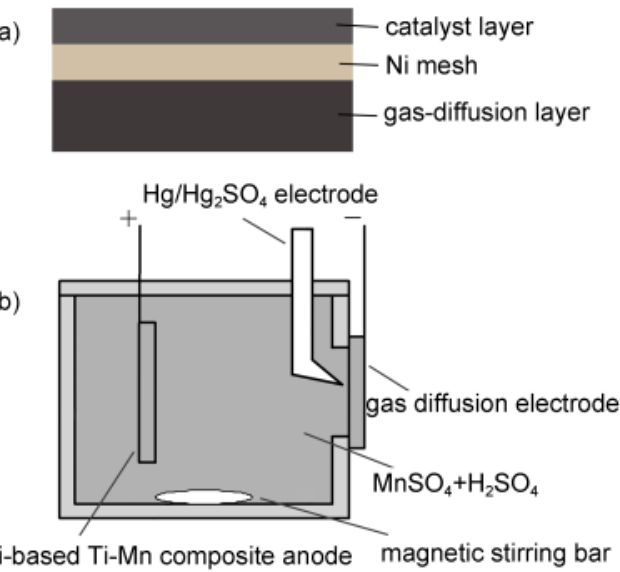

图 1 (a) 气体扩散电极(GDE)及(b)实验装置示意图 ${ }^{1,2}$

Fig.1 Schematic of (a) gas diffusion electrode (GDE) and (b) experimental device $\mathrm{e}^{1,2}$

后气体扩散电极发生的物相变化, 实验条件为 $\mathrm{Cu}$ $K_{a}$ 线, 镍滤波, 扫描速率为 $2\left(^{\circ}\right) \cdot \mathrm{min}^{-1}$, 扫描范围为 $10^{\circ}-90^{\circ}$. 采用德国 Cambridge-S360 扫描电子显微 镜分析气体扩散电极失效前后的形貌及 $\mathrm{Pt}$ 分布. 采 用美国 Trace Northen 能谱仪(EDX)进行表面成分分 析.

\section{3 结果与讨论}

\section{1 气体扩散电极的稳定性及寿命研究}

电解过程中阴、阳两极间的电位差称为槽电 压. 即:

$E=E_{1}+\eta^{+}+\eta^{-}+E_{2}+E_{3}$

式中 $E_{1}$ 为理论分解电压; $\eta^{+}$为阳极过电位; $\eta^{-}$为阴极 过电位; $E_{2}$ 为极间电解液电位; $E_{3}$ 为槽外导体分摊电 位.

气体扩散电极在电流密度为 $100 \mathrm{~A} \cdot \mathrm{m}^{-2}$ 、电解温 度为 $70{ }^{\circ} \mathrm{C}$ 、电解液中 $\mathrm{MnSO}_{4} \cdot \mathrm{H}_{2} \mathrm{O}$ 浓度为 $120 \mathrm{~g} \cdot \mathrm{L}^{-1}$ 、 $\mathrm{H}_{2} \mathrm{SO}_{4}$ 浓度为 $30 \mathrm{~g} \cdot \mathrm{L}^{-1}$ 时槽电压及阴极电位随时间 的变化曲线如图 2 所示.

从图 2 中可以看出, 电解初期槽电压比较稳定, 这个过程大约持续 $35 \mathrm{~h}$, 随后槽电压迅速上升 0.6$0.7 \mathrm{~V}$, 此后槽电压经过一个长时间的稳定期后又迅 速升高; 电解初期阴极电位略有上升, 这个过程大 约持续 $25 \mathrm{~h}$, 幅度不超过 $0.1 \mathrm{~V}$, 电解大约 $375 \mathrm{~h}$ 后阴 极电位迅速下降; 大约电解 $35 \mathrm{~h}$ 后阳极电位迅速上 升, 此后阳极电位基本稳定. 对比槽电压和阳极电 位曲线可以发现电解大约 $35 \mathrm{~h}$ 时槽电压的升高与 阳极电位的升高同步, 且变化幅度相近, 初步推测 槽电压的升高是由于阳极沉积了 EMD; 对比槽电压 


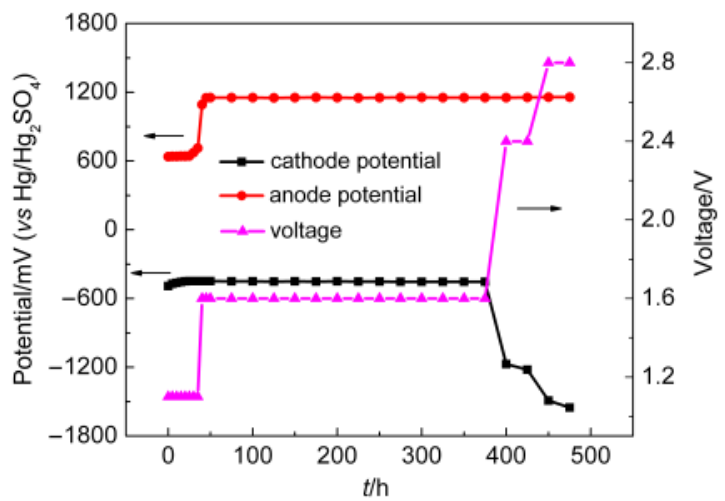

图 2 阴极电位、阳极电位及槽电压随时间的变化曲线

Fig.2 Changing curves of cathode potential, anode potential, and voltage $v$ s time

$C\left(\mathrm{MnSO}_{4} \cdot \mathrm{H}_{2} \mathrm{O}\right)=120 \mathrm{~g} \cdot \mathrm{L}^{-1}, C\left(\mathrm{H}_{2} \mathrm{SO}_{4}\right)=30 \mathrm{~g} \cdot \mathrm{L}^{-1}$, $I=100 \mathrm{~A} \cdot \mathrm{m}^{-2}, T=70^{\circ} \mathrm{C}$

和阴极电位曲线可以发现, 槽电压的再次升高与阴 极电位的降低同步, 且变化幅度相近, 同时观察到 阴极析出大量气泡, 初步推测槽电压再次升高的原 因是阴极失效. 电解初期, 阴极电位略微上升的原 因主要是溶液逐渐渗透到催化层中与催化剂充分 接触, 形成足够多的三相界面后趋于稳定.

从公式(1)槽电压的组成可知, 其中理论分解电 压、极间电解液电压降和电解槽外导体分摊电位不 变, 影响槽电压的因素主要是阴极电位和阳极电 位. 电解初期阴极电位变化幅度不超过 $0.1 \mathrm{~V}$, 说明 阴极电位的变化不是槽电压升高的原因. 为了找到 电解初期槽电压升高的原因, 我们做了平行实验, 如图 3 所示.

图 3 中 $a 、 b$ 曲线为平行试样, $a$ 曲线中阳极产物 不经过处理, $\mathrm{b}$ 曲线中分别在 65 和 $135 \mathrm{~h}$ 时将阳极产 物剥离, 然后将处理过的阳极板再次放入电解槽

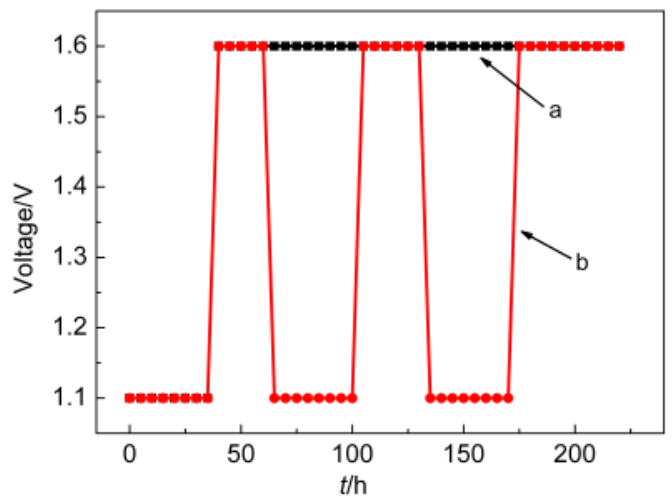

图 3 槽电压随时间的变化曲线

Fig.3 Changing curve of cell voltage $v s$ time

(a) anode product without peel, (b) anode product with peel; $C\left(\mathrm{MnSO}_{4} \cdot \mathrm{H}_{2} \mathrm{O}\right)=120 \mathrm{~g} \cdot \mathrm{L}^{-1}, C\left(\mathrm{H}_{2} \mathrm{SO}_{4}\right)=30 \mathrm{~g} \cdot \mathrm{L}^{-1}$, $I=100 \mathrm{~A} \cdot \mathrm{m}^{-2}, T=70^{\circ} \mathrm{C}$
中. 从图中可以看出, 去除其表面沉积的 EMD 后槽 电压降至初始值, 电解大约 $35 \mathrm{~h}$ 后槽电压再次升高 $0.5 \mathrm{~V}$. 与图 2 中所得结论一致, 槽电压初期升高的原 因是电解过程中阳极表面沉积 EMD, 由于 EMD 导 电性差, 槽电压升高主要是用于克服阳极表面与新 生二氧化锰之间的界面能.

\section{2 气体扩散电极电化学行为的研究}

在制备 EMD 时, GDE 上主要发生氧还原反应 和氢还原反应. 当 $p_{\mathrm{O}_{2}}=100 \mathrm{~Pa} 、 p_{\mathrm{H}_{2}}=100 \mathrm{~Pa} 、 T=70^{\circ} \mathrm{C}$ 、 $\mathrm{pH}=0.7$ 时, $\mathrm{O}_{2}+4 \mathrm{H}^{+}+4 \mathrm{e}=2 \mathrm{H}_{2} \mathrm{O}(\varphi=1.143 \mathrm{~V}), 2 \mathrm{H}^{+}+2 \mathrm{e} \longrightarrow$ $\mathrm{H}_{2}(\varphi=-0.046 \mathrm{~V})$, 两种阴极反应的理论电位差为 $1.19 \mathrm{~V}$ 左右. 当 GDE有足够的三相界面和充足的氧 气时, 阴极主要发生氧还原反应, 由于氧电极过程 总伴随有较大的过电位, 氧还原反应的分解电压要 远低于 $1.143 \mathrm{~V}^{21}$

为了找到电解后期槽电压升高及阴极电位降 低的原因, 我们将未使用的 GDE、图 2 中所用的 GDE (电解 $500 \mathrm{~h}$ ) 及无氧条件时的 GDE 极化曲线进 行对比, 如图 4 所示. 测试条件为 $C\left(\mathrm{MnSO}_{4} \cdot \mathrm{H}_{2} \mathrm{O}\right)=$ $120 \mathrm{~g} \cdot \mathrm{L}^{-1}, C\left(\mathrm{H}_{2} \mathrm{SO}_{4}\right)=30 \mathrm{~g} \cdot \mathrm{L}^{-1}, T=70{ }^{\circ} \mathrm{C}$, 扫描速率为 $5 \mathrm{mV} \cdot \mathrm{s}^{-1}$, 参比电极为 $\mathrm{Hg} / \mathrm{Hg}_{2} \mathrm{SO}_{4}$.

图 4 中 $\mathrm{a}$ 曲线为气体扩散电极上氧还原反应过 程的阴极极化曲线, 由于控制因素的不同, 这条曲 线可以分为四个部分. ${ }^{22}$ 曲线 $\mathrm{EF}$ 段, 阴极过程在不大 的电流密度下进行, 且阴极表面氧的供应相对比较 充足, 阴极过程的速度由氧离子化过电位所控制; 曲线 $\mathrm{FG}$ 段, 随着电流密度的增大, 由于浓度极化的 出现, 阴极过程的速度将与氧的离子化和氧的扩散 过程都有关, 阴极过程的速度由氧的离子化反应和

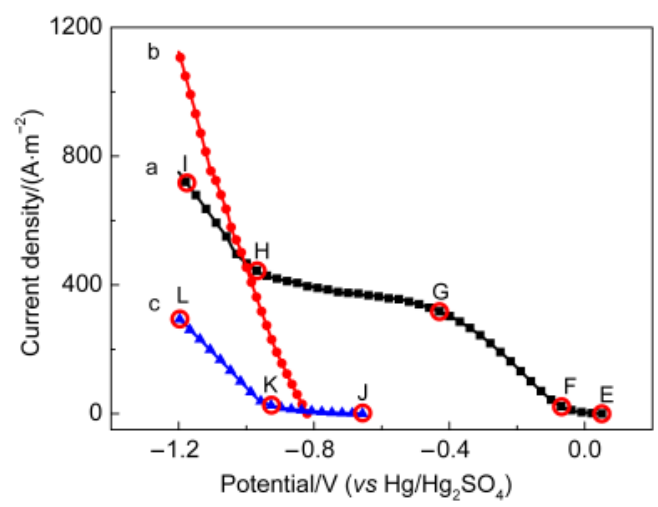

图 4 气体扩散电极的极化曲线

Fig.4 Polarization curves of gas diffusion electrode (a) untapped GDE; (b) in the absence of oxygen; (c) after $500 \mathrm{~h}$ of electrolysis 
氧的扩散混合控制; ${ }^{23}$ 曲线 $\mathrm{GH}$ 段, 随着电流密度的 增大, 由于扩散过程的阻滞而引起极化不断增加, 极化曲线开始很平缓地上升, 在这种情况下, 因为 电极电位大大地向负方向移动, 氧离子化的电极反 应已经被大大活化, 只要氧一到达电极表面立即被 还原, 此时整个阴极过程的速度仅仅由氧的扩散过 程所控制; 曲线 HI 段, 阴极过程由氧去极化和氢去 极化共同组成, 电极上发生析氢反应. 从 $\mathrm{a}$ 曲线可以 看出, 当电流密度为 $100 \mathrm{~A} \cdot \mathrm{m}^{-2}$ 时, 阴极过程由氧的 离子化反应与氧的扩散过程混合控制, 此时提高温 度和氧的浓度都可以减少阴极极化. 图 4 中 $\mathrm{b}$ 曲线 为 GDE 在无氧条件下的极化曲线, 由于没有氧提 供, 此时 GDE 只作为普通析氢阴极. ${ }^{22,24}$ 对比曲线 $\mathrm{a}$ 、 $\mathrm{b}$ 可以看出, 电极电位负于 $-0.8 \mathrm{~V}$ 时, 电极反应不完 全为氧还原反应. 图 4 中 $\mathrm{c}$ 曲线为 GDE在电解 $500 \mathrm{~h}$ 后的极化曲线, 曲线 JK段, 由于 GDE 被破坏, 溶液 浸入电极中, 大量气液固三相界面被淹没, 没有足 够的氧气提供, 阴极过程由氧的扩散过程所控制; 曲线 KL 段, 当电流密度略微增大时, 极化曲线就开 始很陡的上升, 阴极过程由氧去极化和氢去极化共 同组成, 从图 $4 \mathrm{c}$ 曲线中可以看出, 当电流密度为 $100 \mathrm{~A} \cdot \mathrm{m}^{-2}$ 时, 阴极过程由氧去极化和氢去极化共同
组成, 由于气液固三相界面被淹没没有氧气提供, 此时主要发生析氢反应, GDE 失效. 在实验过程中 观察到阴极冒出大量气泡也验证了这一点.

\section{3 气体扩散电极失效行为的研究}

我们进行了平行实验, 将不同时间节点的气体 扩散电极横截面扫描电子显微镜(SEM) 图及 $\mathrm{Mn}^{2+}$ 在 界面区域的分布与含量进行了对比, 如图 5 所示.

图 5(a)为未使用的 GDE; 图 5(b) 为电解 $100 \mathrm{~h}$ 后, 电解液逐渐渗入催化层中, 随着催化层的深入, $\mathrm{Mn}^{2+}$ 含量减少; 图 5(c) 为电解 $200 \mathrm{~h}$ 后, 靠近镍网的 催化层 $\mathrm{Mn}^{2+}$ 含量最高, 说明此时电解液完全浸没催 化层; 图 5(d) 为电解 $300 \mathrm{~h}$ 后, 镍网层和催化层中 $\mathrm{Mn}^{2+}$ 含量相当, 且明显高于气体扩散层, 说明此时电 解液充分与镍网接触; 图 5(e)为电解 $400 \mathrm{~h}$ 后, 镍网 被部分溶解, 催化层有大量裂纹和孔洞; 图 5(f) 为电 解 $500 \mathrm{~h}$ 后, 镍网已经完全溶解, 催化层与扩散层分 离, 电解液完全浸没电极. 随着电解时间的延长, $\mathrm{Mn}^{2+}$ 逐渐渗透到催化层、镍网层和气体扩散层中, 镍 网层的溶解使得催化层和气体扩散层分离, 电解液 完全浸没 GDE, 最后电解液穿透电极.

为了找到气体扩散电极失效的原因, 对比了未 使用的 GDE 和图 2 中所用 GDE(电解 $500 \mathrm{~h}$ ) 的催化
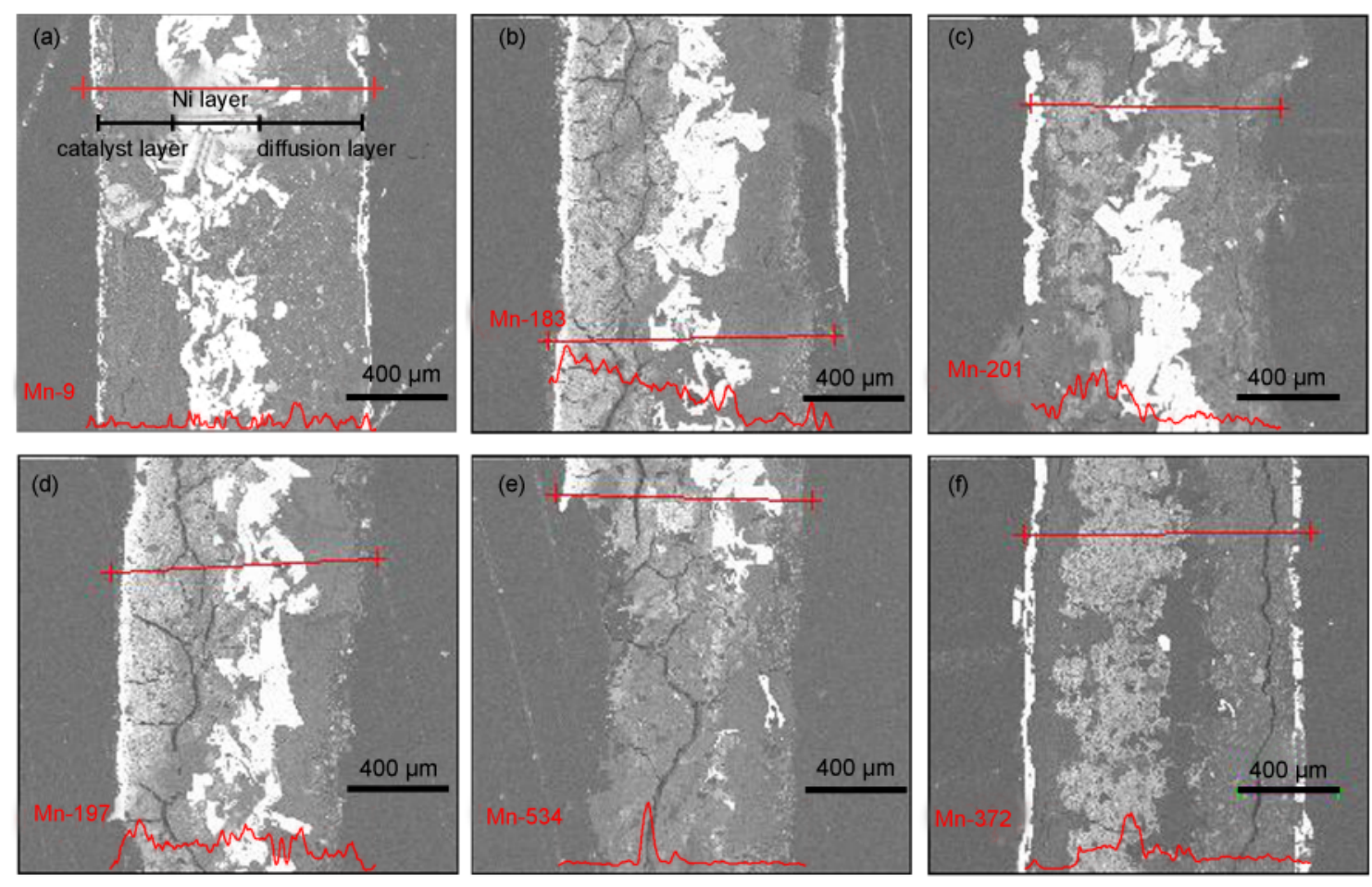

图 5 电解后气体扩散电极横截面的扫描电镜(SEM)图及 $\mathrm{Mn}^{2+}$ 线分析图

Fig.5 Scanning electron microscopy (SEM) images and line analyses of the $\mathrm{Mn}^{2+}$ cross section of GDE after electrolysis $t / \mathrm{h}:$ (a) 0 , (b) 100 , (c) 200 , (d) 300 , (e) 400 , (f) $500 ; C\left(\mathrm{MnSO}_{4} \cdot \mathrm{H}_{2} \mathrm{O}\right)=120 \mathrm{~g} \cdot \mathrm{L}^{-1}, C\left(\mathrm{H}_{2} \mathrm{SO}_{4}\right)=30 \mathrm{~g} \cdot \mathrm{L}^{-1}, I=100 \mathrm{~A} \cdot \mathrm{m}^{-2}, T=70{ }^{\circ} \mathrm{C}$ 


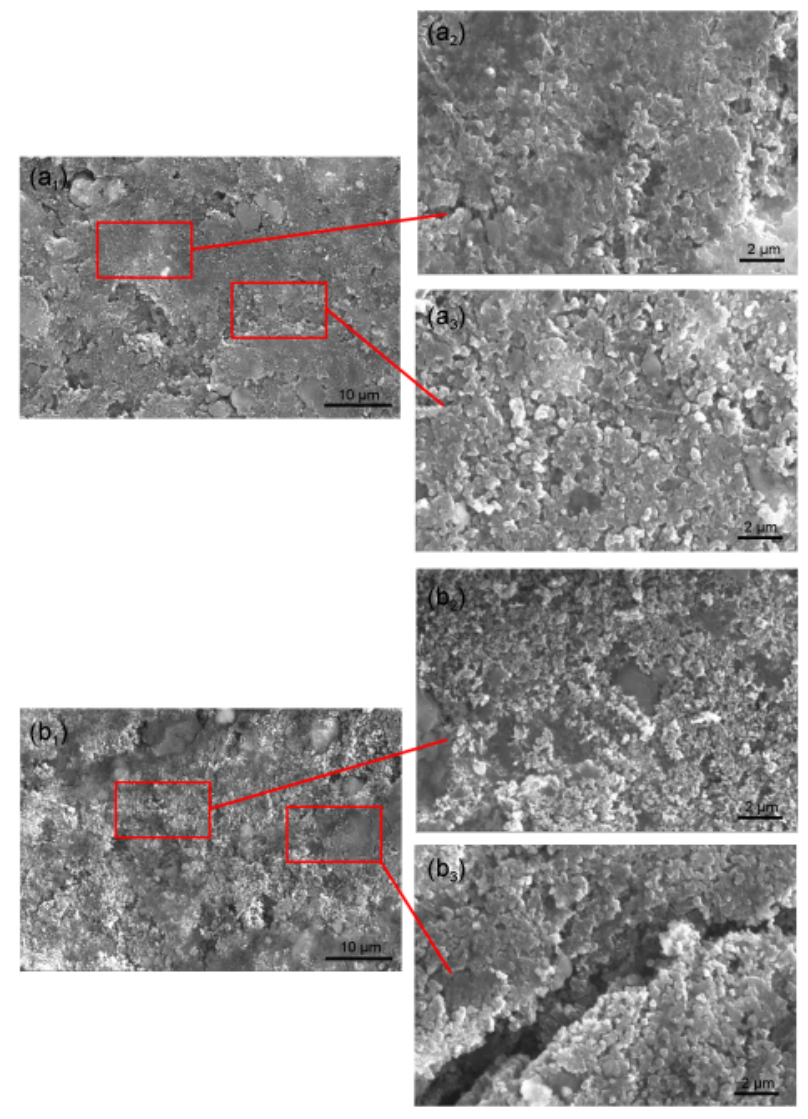

图 6 气体扩散电极催化层表面的 SEM 图

Fig.6 SEM images of the surface of the catalyst layer of GDE (signal A=SE1)

$\left(a_{1}, a_{2}, a_{3}\right)$ untapped GDE; $\left(b_{1}, b_{2}, b_{3}\right)$ after $500 \mathrm{~h}$ of electrolysis; $C\left(\mathrm{MnSO}_{4} \cdot \mathrm{H}_{2} \mathrm{O}\right)=120 \mathrm{~g} \cdot \mathrm{L}^{-1}, C\left(\mathrm{H}_{2} \mathrm{SO}_{4}\right)=30 \mathrm{~g} \cdot \mathrm{L}^{-1}$, $I=100 \mathrm{~A} \cdot \mathrm{m}^{-2}, T=70^{\circ} \mathrm{C}, t=500 \mathrm{~h}$

层表面形貌, 如图 6 所示.

图 6(a $\left.a_{1}\right)$ 为未使用的 GDE 催化层放大 3000 倍的 SEM 图, 催化层表面布满了 PTFE网络结构, 将粉末 颗粒紧密粘结在一起, 并形成许多微小的孔隙, 图 6 $\left(a_{2}\right)$ 为较薄区域的放大图, 图 $6\left(a_{3}\right)$ 为较厚区域的放大 图. 图 6(b $\left.b_{1}\right)$ 为催化层使用 $500 \mathrm{~h}$ 后放大 3000 倍的 SEM 图, 催化层表面颗粒比较松散, 图 6(b $\left.\mathrm{b}_{2}\right)$ 为 PTFE 网络结构破坏的区域放大图, 图 6(b $\left.\mathrm{b}_{3}\right)$ 为裂纹区域放 大图, 可以看到 PTFE 网络结构被破坏, 且有孔洞和 裂缝出现, 原因可能是催化层表面 PTFE 部分溶解, 使得网络结构被破坏, 同时孔隙中有硫酸锰析出, 破坏了催化层结构, 从而形成裂缝.

从图 5 和图 6 中我们可以推测, 电极失效的原 因是 GDE 催化层的PTFE 网络结构被破坏, 从而形 成大量孔洞和裂缝, 电解液充分与镍网接触, 加速 了镍网的溶解. 镍网的溶解和三相界面的减少使得 氧气不能参与反应, 电极更容易发生析氢反应, 加
速了电极的破坏. 同时也说明了图 2 中, 槽电压再次 升高的原因是阴极失效.

我们将未使用的 GDE 和图 2 中所用 GDE(电解 $500 \mathrm{~h}$ )的催化层成分、催化层表面形貌进行了对比, 如图 7 和图 8 所示.

图 7 为气体扩散电极未使用和电解 $500 \mathrm{~h}$ 后 $(I=$ $100 \mathrm{~A} \cdot \mathrm{m}^{-2} 、 T=70{ }^{\circ} \mathrm{C}$ 、电解液中 $\mathrm{MnSO}_{4} \cdot \mathrm{H}_{2} \mathrm{O}$ 浓度为 $120 \mathrm{~g} \cdot \mathrm{L}^{-1} 、 \mathrm{H}_{2} \mathrm{SO}_{4}$ 浓度为 $30 \mathrm{~g} \cdot \mathrm{L}^{-1}$ )催化层的 XRD 图. 图 7 中曲线 $\mathrm{a}$ 为未使用的 GDE 催化层的 XRD 图, 图 谱中主要为聚四氟乙烯(PTFE)和 $\mathrm{C}$ 的衍射峰和较弱 的 $\mathrm{Pt}$ 衍射峰; 图 7 中曲线 $\mathrm{b}$ 为图 2 中电解使用 $500 \mathrm{~h}$ 后 GDE 催化层的 XRD 图谱, 图中主要为 PTFE、C、 $\mathrm{Ni}$ 和 Pt 的衍射峰. 对比曲线 $\mathrm{a} 、 \mathrm{~b}$ 可以发现, $\mathrm{Pt}$ 的衍射 峰明显增强, 这是因为碳载铂只占催化层的 5\%(w), 而碳载铂中 Pt 颗粒约为 $10 \mathrm{~nm}$, 由于 Pt 颗粒尺寸细 小, 颗粒边缘不规则的晶体结构对 XRD 衍射峰贡献 比较大, 使得 $\mathrm{Pt}$ 衍射峰很弱; 随着电解反应的发生, $\mathrm{Pt}$ 颗粒不断团聚、长大, 颗粒边缘不规则的晶体结 构对 XRD 衍射峰贡献减小, 而颗粒内部规则的晶体 结构对 XRD 衍射峰贡献增大, 使得 Pt 衍射峰明显增 强. 曲线 $\mathrm{a}$ 中没有镍的衍射峰, 而曲线 $\mathrm{b}$ 中出现了镍 的衍射峰, 未使用的 GDE 催化层中不含镍, 而电解 液中也没有镍离子, 可以推测在电极反应过程中, 电解液浸入镍网层中, 使得镍网溶解. 部分溶解的 镍沉积在催化层上, 因而镍衍射峰来自镍网层的溶 解, 与图 5(e)、5(f)结论一致.

图 8 为气体扩散电极未使用和电解 $500 \mathrm{~h}$ 后 $(I=$ $100 \mathrm{~A} \cdot \mathrm{m}^{-2} 、 T=70{ }^{\circ} \mathrm{C}$ 、电解液中 $\mathrm{MnSO}_{4} \cdot \mathrm{H}_{2} \mathrm{O}$ 浓度为 $120 \mathrm{~g} \cdot \mathrm{L}^{-1} 、 \mathrm{H}_{2} \mathrm{SO}_{4}$ 浓度为 $30 \mathrm{~g} \cdot \mathrm{L}^{-1}$ ) 催化层的 SEM 图.

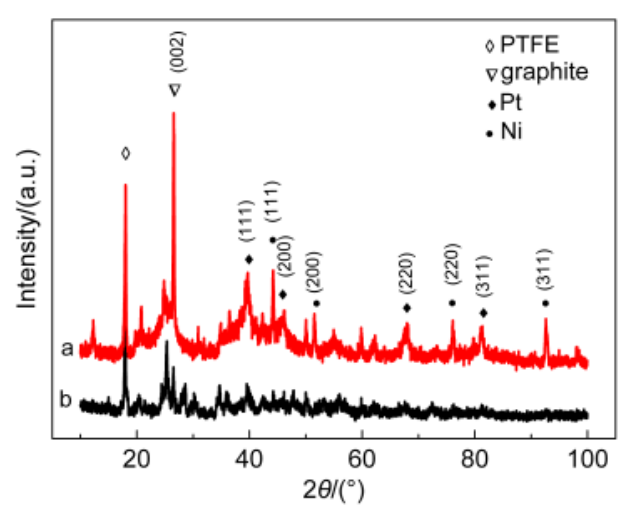

图 7 气体扩散电极催化层的 XRD

Fig.7 XRD patterns of the catalyst layer of GDE

(a) untapped GDE, (b) after $500 \mathrm{~h}$ of electrolysis; $I=100 \mathrm{~A} \cdot \mathrm{m}^{-2}$; $T=70{ }^{\circ} \mathrm{C} ; C\left(\mathrm{MnSO}_{4} \cdot \mathrm{H}_{2} \mathrm{O}\right)=120 \mathrm{~g} \cdot \mathrm{L}^{-1} ; C\left(\mathrm{H}_{2} \mathrm{SO}_{4}\right)=30 \mathrm{~g} \cdot \mathrm{L}^{-1}$ 

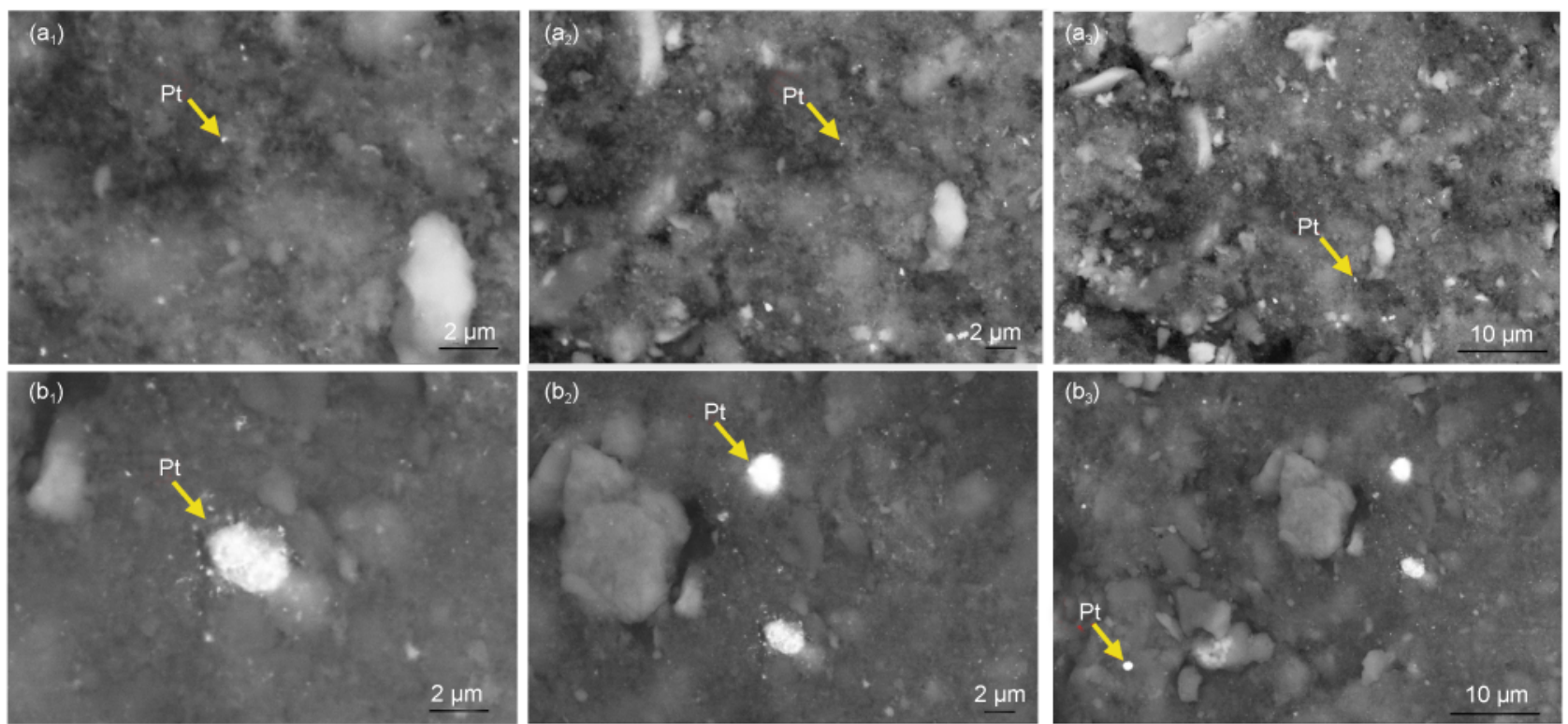

图 8 气体扩散电极催化层的 SEM 图

Fig.8 SEM image of the catalyst layer of GDE (signal A=QBSD)

$\left(a_{1}, a_{2}, a_{3}\right)$ untapped GDE, $\left(b_{1}, b_{2}, b_{3}\right)$ after $500 \mathrm{~h}$ of electrolysis;

$C\left(\mathrm{MnSO}_{4} \cdot \mathrm{H}_{2} \mathrm{O}\right)=120 \mathrm{~g} \cdot \mathrm{L}^{-1}, C\left(\mathrm{H}_{2} \mathrm{SO}_{4}\right)=30 \mathrm{~g} \cdot \mathrm{L}^{-1}, I=100 \mathrm{~A} \cdot \mathrm{m}^{-2}, T=70{ }^{\circ} \mathrm{C}, t=500 \mathrm{~h}$

图 $8\left(a_{1}, a_{2}, a_{3}\right)$ 为未使用的气体扩散电极催化层, 高度 分散的、亮度最高的白色颗粒为 Pt 颗粒, 图中 Pt 颗 粒分散均匀, 无明显的团聚; 图 $8\left(b_{1}, b_{2}, b_{3}\right)$ 为电解 $500 \mathrm{~h}$ 后气体扩散电极的催化层, 可以明显看到 $\mathrm{Pt}$ 颗粒发生团聚且分散不均匀. 对比图 8(a)、8(b)可以 发现, 随着电解时间的增加, Pt 颗粒不断团聚、长 大. 同时也验证了 XRD 中, Pt 衍射峰增强的原因是 $\mathrm{Pt}$ 颗粒的团聚.

从气体扩散电极使用前后催化层的 $\mathrm{XRD}$ 和 SEM 对比图可以推测, 催化剂的团聚使得氧还原反 应的活性面积减小, 阴极极化增大, 当阴极过程由 氧去极化和氢去极化共同组成时, 阴极开始析出氢 气, 氢气的析出加速了催化层的 PTFE 网络结构的 破坏, 从而加速了电极的失效, Pt 的团聚是造成电 极失效的主要原因. 催化剂的团聚和脱落也是气 体扩散电极在碱性和弱酸性条件下失效的主要 原因. ${ }^{25,26}$

\section{4 结 论}

(1) 寿命实验表明, 本文中采用的电极制备工艺 基本稳定, 气体扩散电极在强酸性 $\mathrm{MnSO}_{4}-\mathrm{H}_{2} \mathrm{SO}_{4}$ 电 解液中具有一定的稳定性, 寿命可达 $400 \mathrm{~h}$. 平行实 验表明, 电解过程中槽电压第一次升高的原因是阳 极沉积了 EMD, 为了克服阳极表面与新生二氧化锰 之间的界面能; 槽电压再次升高的原因是阴极失
效, 此时阴极主要发生析氢反应.

(2) 电化学实验表明, 电流密度为 $100 \mathrm{~A} \cdot \mathrm{m}^{-2}$, 气 体扩散电极未失效时阴极过程的速度由氧的离子 化反应和氧的扩散混合控制; 气体扩散电极失效 后, 阴极过程由氧去极化和氢去极化共同组成, 由 于三相界面消失, 空气中的氧气不能参与反应, 此 时主要发生析氢反应.

(3) 气体扩散电极使用前后催化层的表面形貌 图表明, 电解过程中 PTFE 部分溶解, PTFE 网络结 构被破坏, 且有孔洞和裂缝出现; 不同时间节点的 气体扩散电极横截面 SEM 图及 $\mathrm{Mn}^{2+}$ 在界面区域的 分布与含量图表明, 电解液逐渐浸入电极中, 镍网 层溶解, 催化层与扩散层分离, 最终导致电极漏液. PTFE网络结构被破坏和镍网层的溶解是 GDE 失效 的原因之一。

(4) 气体扩散电极使用前后催化层的 XRD 和 SEM 对比图表明, 电解过程中 Pt 发生了团聚, 电极 中的镍网层发生了溶解, $\mathrm{Pt}$ 的团聚降低了电极的电 催化活性是造成GDE失效的主要原因.

\section{References}

(1) Meng, H. M.; Guo, L.; Zhang, H.; Sun, D. B.; Yu, H. Y.; Fan, Z. S.; Wang, X. D. An Energy-Saving Method for Preparing Electrolytic Manganese Dioxide. CN Patent ZL201010578846.5, 2011-4-27. [孟惠民, 郭 琳, 张 慧, 孙 冬柏, 俞红英, 㚞自栓, 王旭东. 一种节能型电解二氧化锰的制 
备方法: 中国, ZL201010578846.5[P]. 2011-4-27.]

(2) Zhang, H.; Meng, H.; Guo, L.; Yu, H. Y.; Sun, D. B. China's Manganese Industry 2013, 31 (1), 27. [张＼cjkstart慧, 孟惠民, 郭 琳, 俞红英, 孙冬柏. 中国锰业, 2013, 31 (1), 27.]

(3) Chen, S.; Xiao, Z. B.; Liu, J. B.; Yin, Y. L. Journal of Jishou University (Natural Sciences Edition) 2009, 30 (3), 86. [陈上, 肖卓炳, 刘建本, 尹艳林. 吉首大学学报(自然科学 版), 2009, 30 (3), 86.]

(4) Fukada, S. Energ. Convers. Manage 2001, 42 (9), 1121. doi: 10.1016/S0196-8904(00)00124-2

(5) Ramousse, J.; Deseure, J.; Lottin, O.; Didierjean, S.; Maillet, D. J. Power Sources 2005, 145 (2), 416. doi: 10.1016/j.jpowsour. 2005.01.067

(6) Grigoriev, S. A.; Kalinnikov, A. A.; Kuleshov, N. V.; Millet, P. Int. J. Hydrog. Energy 2013, 38 (20), 8557. doi: 10.1016/j. ijhydene.2012.10.056

(7) Chabi, S.; Kheirmand, M. Appl. Surf. Sci. 2011, 257 (24), 10408. doi: 10.1016/j.apsusc.2011.06.104

(8) Moussallem, I.; Jörissen, J.; Kunz, U.; Pinnow, S.; Turek, T. J. Appl. Electrochem. 2008, 38 (9), 1177. doi: 10.1007/s10800008-9556-9

(9) Pinnow, S.; Chavan, N.; Turek, T. J. Appl. Electrochem. 2011, 41 (9), 1053. doi: 10.1007/s10800-011-0311-2

(10) Marini, S.; Salvi, P.; Nelli, P.; Pesenti, R.; Villa, M.; Kiros, Y. Int. J. Hydrog. Energy 2013, 38 (26), 11496. doi: 10.1016/j. ijhydene.2013.04.160

(11) Dong, H.; Yu, H. B.; Wang, X.; Zhou, Q. X.; Feng, J. L. Water Res. 2012, 46 (17), 5777. doi: 10.1016/j.watres.2012.08.005

(12) Lee, S. J.; Pyun, S. I. Electrochim. Acta 2007, 52 (23), 6525. doi: 10.1016/j.electacta.2007.04.081

(13) Chhina, H.; Campbell, S.; Kesler, O. J. Power Sources 2008, 179 (1), 50. doi: 10.1016/j.jpowsour.2007.12.105

(14) Chhina, H.; Campbell, S.; Kesler, O. J. Power Sources 2007, 164 (2), 431. doi: 10.1016/j.jpowsour.2006.11.003

(15) Nagai, M.; Yoshida, M.; Tominaga, H. Electrochim. Acta 2007, 53 (2), 1030. doi: 10.1016/j.electacta.2007.09.036
(16) Ma, C. A.; Huang, Y.; Zhu, Y. H.; Chen, Z. Y.; Lin, W. F. CIESC Journal 2009, 60 (10), 2633. [马淳安, 黄 望, 朱英红, 陈赵 扬, 林文锋. 化工学报, 2009, 60 (10), 2633.]

(17) Fu, J. L.; Zhang, X. W.; Lei, L. C. Acta Phys. -Chim. Sin. 2007, 23 (8), 1157. [傅坚亮, 张兴旺, 雷乐成. 物理化学学报, 2007, 23 (8), 1157.] doi: 10.1016/S1872-1508(07)60060-6

(18) Hayashi, K.; Furuya, N. J. Electrochem. Soc. 2004, 151 (3), A354.

(19) Yu, Q. H.; Zhou, M. H.; Lei, L. C. Acta Phys. -Chim. Sin. 2006, 22 (7), 883. [ 郁青红, 周明华, 雷乐成. 物理化学学报, 2006, 22 (7), 883.] doi: 10.3866/PKU.WHXB20060723

(20) Dai, X. F.; Zhen, M. F.; Xu, P.; Shi, J. J.; Ma, C. Y.; Qiao, J. L. Acta Phys. -Chim. Sin. 2013, 29 (8), 1753. [戴先逢, 郑明富, 徐攀, 石晶晶, 马承禺, 乔锦丽. 物理化学学报, 2013, 29 (8), 1753.] doi: 10.3866/PKU.WHXB201306141

(21) Li, D. Electrochemistry Principle; Beijing University of Aeronautics and Astronautics Press: Beijing, 1999; pp 388389. [李 荻. 电化学原理. 北京: 北京航空航天大学出版社, 1999: 388-389.]

(22) Wei, B. M. Metal Corrosion Theory and Application; Chemical Industry Press: Beijing, 2008; pp 94-95, 105-107. [魏宝明. 金属腐蚀理论及应用. 北京: 化学工业出版社, 2008: 94-95, 105-107.]

(23) Sudoh, M.; Arai, K.; Lzawa, Y.; Suzuki, T.; Uno, M.; Tanaka, M.; Hirao, K.; Nishiki, Y. Electrochim. Acta 2011, 56 (28), 10575. doi: 10.1016/j.electacta.2011.06.013

(24) Li, J. B.; Pan, Z. C.; Xiao, C. M.; Zhang, H. H.; Deng, Y. Chemistry \& Bioengineering 2007, 24 (7), 16. [李嘉斌, 潘湛 昌, 肖楚民, 张环华, 邓 仪. 化学与生物工程, 2007, 24 (7), 16.]

(25) Furuya, N.; Aikawa, H. Electrochim. Acta 2000, 45 (25-26), 4251. doi: 10.1016/S0013-4686(00)00557-0

(26) Siracusano, S.; Denaro, T.; Antonucci, V.; Aricò, A. S.; Urgeghe, C.; Federico, F. J. Appl. Electrochem. 2008, 38 (12), 1637. 